\title{
MATTER-ANTIMATTER SEPARATION AND THE ANTIMATTER PROBLEM IN COSMOLOGY
}

\author{
R. OMNÈS \\ Laboratoire de Physique Théorique et Hautes Energies, Bâtiment 211, Université de Paris-Sud, Centre \\ d'Orsay, 91405 - Orsay France
}

\begin{abstract}
I shall report on some work done in France in the last few years concerning the possible existence of antimatter in the Universe. It will be shown that, by taking due account of several physical effects which have been theoretically predicted, one can propose a coherent theory for the origin of matter and galaxies that appears to be quantitatively satisfactory. In this model there should exist as many antigalaxies as galaxies. During the course of this paper, I shall try to make it clear what the basic physical processes are and the present state of their investigation. This discussion will therefore be restricted to the two aforementioned problems, viz., the origin of matter and protogalaxies, leaving aside other astrophysical applications.
\end{abstract}

\section{The Geometrical Background of Cosmology}

As a general rule, we shall consider as a dogma our present knowledge of physics, only trying to investigate some of its consequences without allowing ourselves the freedom of changing the basic law of physics. Only if such a program were to obviously fail, could we feel justified in introducing new ad hoc assumptions. For that reason, general relativity will be taken as a proper overall description of space-time.

The relativistic description of space-time turns out to be particularly simple if one starts from two observed facts:

(i) The Hubble expansion of the Universe

(ii) The existence of a thermal background of radio waves with a temperature of $2.7 \mathrm{~K}$. The radiation is known experimentally to be highly isotropic. We shall not discuss here any alternative interpretation for it.

Furthermore, according to the so-called cosmological principle, it is assumed that our position in the Universe is not privileged so that any other observer located on another galaxy would obtain the same picture of the Universe. Finally, a technical assumption will be to assume that Einstein's cosmological constant is zero.

Starting from these hypotheses, the structure and evolution of space-time have been well known for nearly forty years. The basic tool is provided by Einstein's equations which relate the geometry of space-time to its dynamical content, i.e. in practice to the mass density $\varrho$ and the (radiative) pressure $P$. Isotropy of the background radiation implies that space itself is isotropic. Furthermore, coordinates can be chosen in such a way that there is a universal time $t$ : this means that all co-moving observers (who are essentially located in galaxies) find the same local values of $\varrho$ and $P$ at a given time $t$ and these quantities $\varrho$ and $P$ depend only upon $t$. Basically, this result expresses the fact that isotropy is incompatible with the existence of gradients for the dynamical quantities $\varrho$ and $P$. 
Under these conditions, it turns out that the geometry of spacetime can be characterized by two quantities, the time $t$ and a scale factor $R(t)$ which describes expansion: an increase of $R(t)$ with time corresponds to an increase of the distances between two far-away galaxies in proportion to $R(t)$. (See Figure 1.) The Einstein equations therefore relate the geometry of space-time ( $R$ and $t$ ) to the energy-momentum ( $\varrho$ and $P$ ). The variations of $\varrho$ and $P$ with time are themselves simply related to the scale factor $R$ (since, for instance, $\varrho$ is proportional to $R^{-3}$, whereas, as a consequence of Einstein's equations, $P$ can be shown to be proportional to $R^{-4}$ : this last result is often described by saying that expansion is an isentropic process). Therefore, everything is known and one can solve the equations for $R(t)$ as a function of time.

It is found that $R(t)$ starts from a zero value at some finite time in the past which can be chosen as the origin of time and it increases up to its present value (see Figure 2). The present value of the time can be found by comparing the observed value of the Hubble constant with the theoretically predicted rate of expansion which is given by $(1 / R) \times(\mathrm{d} R / \mathrm{d} t)$. A typical value for this time is of the order of $12 \times 10^{9} \mathrm{yr}$.

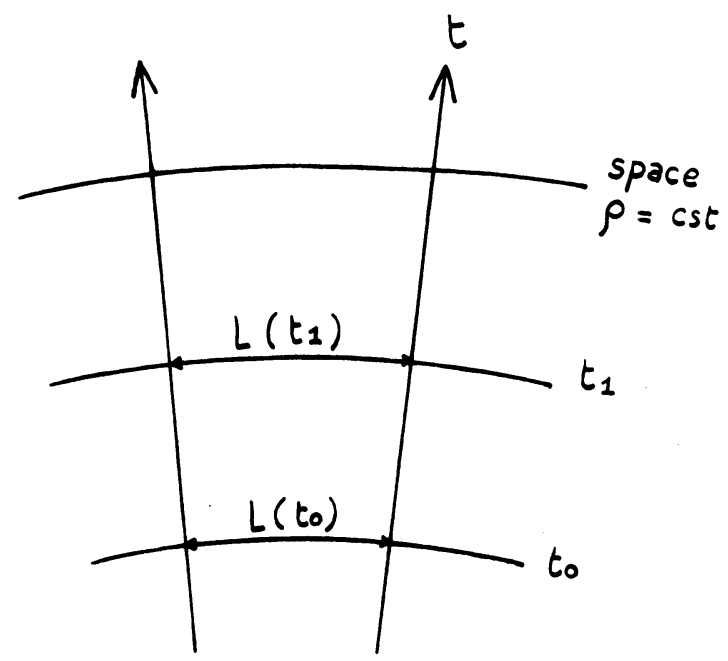

Fig. 1. The expansion of an isotropic Universe.

During the early stages of the Universe (during the first $10^{6} \mathrm{yr}$ or less), its evolution is governed by the thermal radiation it contains. Indeed, the conservation of entropy which is derived from the Einstein equations gives a simple relation between scale and temperature, namely

$$
R T=\text { constant } .
$$

Accordingly, for small values of $R(t)$ or small values of $t$, the temperature can be quite large, so that the contribution of radiation to the mass density and the pressure is dominant. Since these quantities depend only upon the temperature, the only remain- 
ing parameters are $t$ and $T$ which are related by

$$
T(\mathrm{MeV}) \simeq t^{-1 / 2}(\mathrm{sec}) .
$$

From now on we shall stick for simplicity to this specific model. However, it is worth mentioning that most of what we shall say is essentially model-independent: we shall only exploit the existence of locally high temperatures without a real need for the global validity of the isotropic model of the Universe.

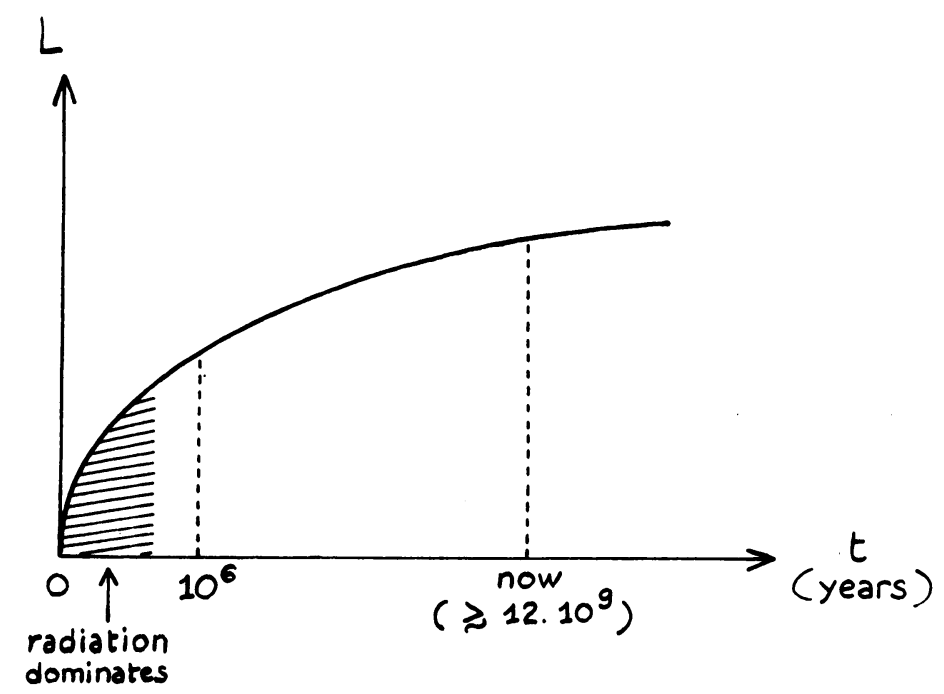

Fig. 2. The scale factor $R$ as a function of time.

\section{What is Thermal Radiation?}

The conventional model of the Universe which has just been described is called the big-bang or the Gamow model. The content of the Universe is made of matter and radiation.

It has been stressed by Gamow that the nature of thermal radiation depends upon temperature: for small enough values of $T$, radiation consists of photons, as we all know. However, when the average thermal energy of photons is large enough to allow the production of electron-positron pairs in photon-photon collisions, one also finds positrons and electrons in radiation. In practice, this occurs when $T$ becomes comparable with the mass energy of electrons, i.e. when $T$ is of the order of $1 \mathrm{MeV}$ or larger. At higher temperatures, other kinds of particles are also present in radiation: for instance, pions can be found when $T$ is of the order of $100 \mathrm{MeV}$, as well as nucleons and antinucleons at higher temperatures.

From Table I let us note the important values $\left(3000 \mathrm{~K}, 10^{6} \mathrm{yr}\right)$ which correspond to the combination of the electrons and protons in matter into atoms. Since the cross section for an interaction between a thermal photon and a neutral atom is much smal- 
ler than for an interaction with a free electron, the coupling between matter and radiation becomes very weak below $3000 \mathrm{~K}$ : in practice, it is found that the photons do not interact any more with matter. Their fate is thereby only to lose energy by red-shift according to (1).

It is very instructive at this stage to compare a few numbers:

(i) The ratio $\eta$ between the densities of nucleons and photons

$$
\eta=\frac{N \text { nucleons }}{N \text { photons }}=\frac{N}{N_{\gamma}}
$$

turns out to be a very fundamental quantity because it stays invariant under expansion. If the Universe were to contain antigalaxies, $\eta$ would be defined as

$$
\eta=\frac{N \text { nucleons }+\bar{N} \text { antinucleons }}{N \text { photons }},
$$

the averages being taken in large regions containing as many antigalaxies as galaxies. The observed value for $\eta$ lies between $10^{-8}$ and $10^{-9}$. This quantity can be taken as a measure of the amount of matter (and antimatter) in the Universe. Sometimes $\eta^{-1}$ is also called the average entropy per baryon.

(ii) One can compare, at any given time, the quantity of nucleons which constitute matter now (their number density varies like $R^{-3}$ ) to the number of nucleons and antinucleons which are part of thermal radiation (their density varies as $T^{\mathbf{3} / 2} e^{-m c^{2} / T}$ ). By now, the second component is negligible, but it is much larger than the first one at $T \geqslant 1 \mathrm{GeV}$ (by a factor $\eta^{-1}$ ). Equality is obtained at $T=30 \mathrm{MeV}$.

TABLE I

The character of radiation at different temperatures

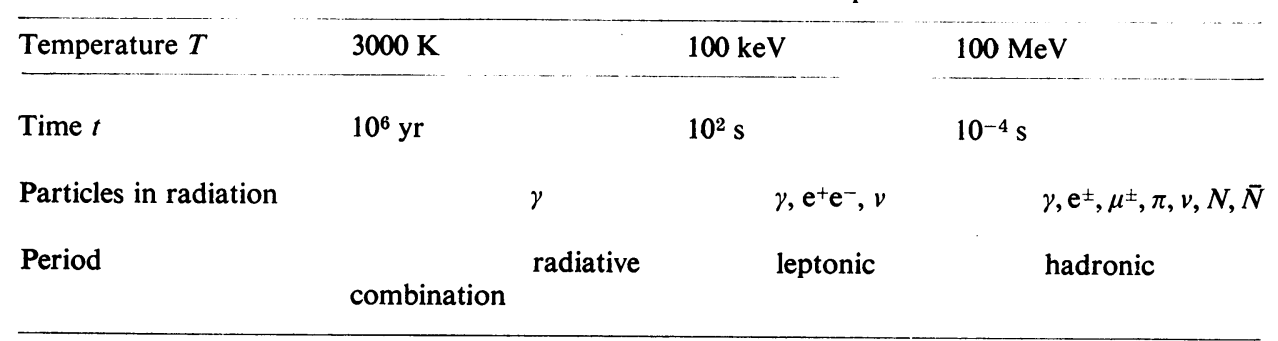

\section{Where Does Matter Come From?}

We are now ready to state the basic assumptions of the antimatter model of the Universe which we intend to investigate. We propose two very simple assumptions:

(i) There exists by now (and always existed) as much antimatter as matter. In other words, the total baryonic number of the Universe is zero.

(ii) The presently observed matter is a relic of the nucleons and antinucleons in the primordial thermal radiation. 
Consideration of a few numbers will help us to envision the problems which must be solved if such a model is to make sense:

We have already noticed that the present amount of matter is comparable to what was found in radiation at a temperature of $30 \mathrm{MeV}$. Therefore, an initial separation of nucleons from antinucleons should have taken place at a higher temperature, i.e. during the hadronic period. But this period is precisely characterized by the fact that the densities of hadrons are large (of the order of the densities in atomic nuclei for temperatures above $200 \mathrm{MeV}$ ), so that strong interactions must play an important role in the behavior of radiation. A natural question to ask is therefore: could it be that strong interactions are responsible for a partial separation of nucleons from antinucleons during the hadronic period?

But even a separation generated by strong interactions cannot be the end of the story. Indeed, the hadronic period ends at a time $t \simeq 10^{-4} \mathrm{~s}$. On the other hand, no physical effect can affect a region with dimensions larger than $c t \simeq 10^{6} \mathrm{~cm}$. Computing the number of nucleons inside such a region at the end of the hadronic period, we find it to be much smaller than the number of nucleons in a galaxy. Since there is unquestionable evidence that normal galaxies are overwhelmingly made of only one type of matter, we must explain how an initial small-scale separation was amplified into a separation of matter from antimatter up to galactic scales.

The fundamental ingredients of our model are specific proposals for each of these mechanisms:

We propose that strong interactions produce a thermodynamic phase transition in thermal radiation at high temperatures. As a result of this transition, nucleons are partially separated from antinucleons. The corresponding effects occurred during the hadronic period.

Once nucleons and antinucleons are separated, annihilation along their boundary generates strong fluid motions. As a result of these motions, matter and antimatter are reorganized into increasingly larger regions of space. This will be called the coalescence effect. It is a corollary of the so-called 'Leidenfrost' mechanism which was first noticed by Alfvén and Klein (1962) and it takes place during the radiative period.

Both effects constitute physical problems that are basically independent of cosmology. The natural framework for the first one is statistical mechanics together with particle physics. For the second one, it is hydrodynamics.

In the following, we shall give our reasons for proposing these effects as well as the present status of their investigation. Later on, cosmological applications to the origin of matter and galaxies will be given.

\section{Approaching Nucleon-Antinucleon Separation}

We shall first describe the general behavior of nucleon-antinucleon separation for the sake of orientation.

It is a phase separation effect. To understand what that means, let us compare it with the liquid-vapor phase transition. In Figure 3, one has plotted the critical curve 
for liquid-vapor transition in a graph where the coordinates are density and temperature. The curve appearing in Figure 3 is the critical curve. For values of the density and temperature falling in the shaded area, liquid and vapor are separated in different regions of space. The size and shape of these regions depend upon the detailed history of the system (the time allowed since separation, for instance), but their respective volumes are completely determined by equilibrium.

In Figure 4, a similar plot has been made for the nucleon-antinucleon separation effect. The coordinates are the temperature and the baryonic density

$$
B=N-\bar{N} \text {. }
$$

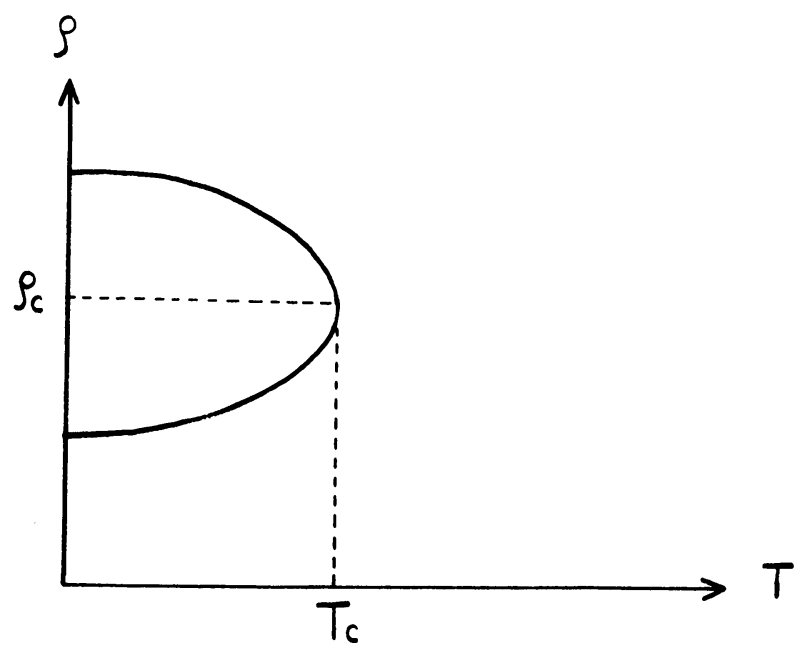

Fig. 3. Phase transition diagram for the liquid-vapor system.

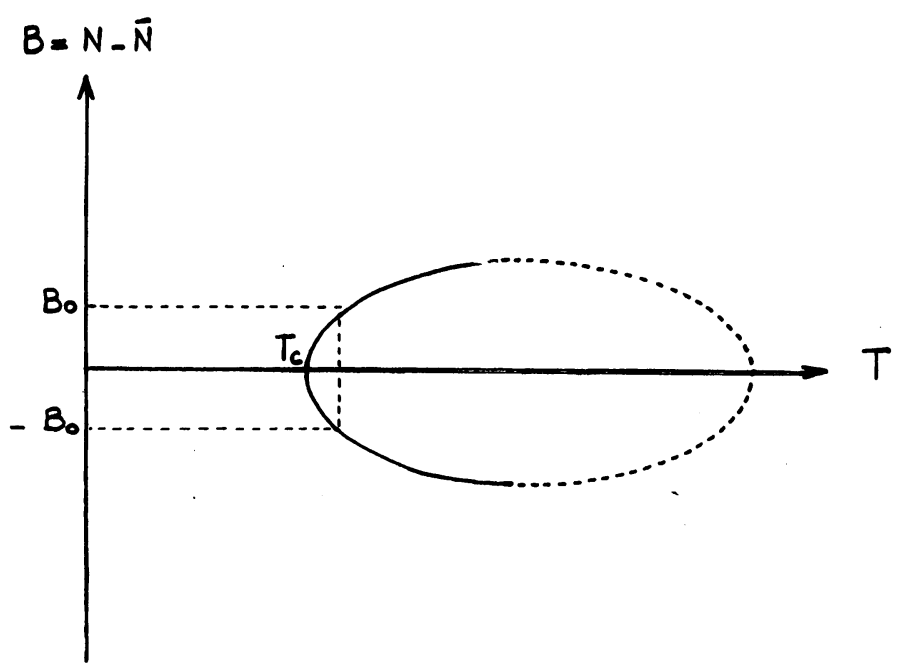

Fig. 4. Phase transition diagram for nucleon-antinucleon separation. 
Since $B$ is allowed to be different from zero, the system under consideration here is a gas of hadrons (for instance, an ordinary gas of matter) which is heated at extremely high temperatures. Here again, we have a critical curve and a critical temperature (which will be found later on to be of the order of $350 \mathrm{MeV}$ ). In the shaded area, the system consists of nucleons and antinucleons which are in spatially distinct regions. More exactly, there are regions with an excess $B_{0}$ of baryonic density or an excess $-B_{0}$ of antibaryonic density. The size of these regions depends upon the history of the system, but their volumes are completely specified by the equilibrium conditions.

The only important difference between the two cases is that, for the liquid-vapor system, the critical curve is convex towards high temperatures, whereas the reverse is true for the nucleon-antinucleon system. This can be understood as follows: separation is basically an effect which takes place at high densities and therefore at high temperatures since there are more and more nucleons and antinucleons in the system as the temperature increases. The shape of the critical curve tells us that the ordering effect due to high density is stronger than the disordering effect due to thermal motion.

It might be that the critical curve closes down at a higher temperature (see the dotted line) But this would be a region of densities and thermal energies where our knowledge of particle physics is still insufficient, so that we shall not discuss it.

Why is there a phase transition? We shall see that the basic reasons are the following:

(i) Mesons $(\pi, \varrho, \omega, \eta)$ are assumed to be nucleon-antinucleon bound states, as first suggested by Fermi and Yang.

(ii) These mesonic bound states are part of thermal radiation, so that the free energy $F$ is a stationary function of their number, i.e., for instance

$$
\frac{\partial F}{\partial N_{\pi}}=0
$$

It is interesting to note that, whereas bound states have been considered in statistical mechanics (for instance, molecules are bound states of atoms), condition (6) is never satisfied in the systems which have been met in the laboratory. In fact, the behavior of nucleons and antinucleons in thermal radiation is the simplest example where these two conditions are satisfied, so that it is worth considering with an open mind.

The task of investigating the thermodynamical behavior of nucleons and antinucleons is a rather formidable one: neither strong interaction physics nor the theory of phase transitions are easy subjects. Obviously, we must resort to highly idealized models. However, it is gratifying that there are by now two such models which both give a positive answer for the existence of the phase transition. Both together give strong support to the idea since the first one, by idealizing the particle physics, leads to a rigorous analysis of the statistical aspects while the second one is probably as good particle physics as can be done presently, approximations being made in the statistical treatment.

\section{A First Model}

We shall first offer a model in which statistical mechanics can be treated rigorously, 
while particle physics is strongly simplified. This model has the advantage of exhibiting the origin of the separation effect more clearly.

Let us treat the system of nucleons and antinucleons as a lattice gas, which means that the particles are located in the cells of a cubic lattice, the size of which is $2 r_{0}$. (See Figure 5.) For $r_{0}$, we shall take the radius of a $N-\bar{N}$ bound state (assuming that such a notion is meaningful). Nucleons and antinucleons can be distributed among the cells but, when a nucleon is already present in a cell, the probability that an antinucleon in the same cell remains a free particle, rather than forming a bound state, is of the order of

$$
P_{0}=e^{-m c^{2} / T} \text {. }
$$

On the other hand, according to (6), the bound states do not contribute to the free energy and may be forgotten. The model then consists in considering the small quantity $P_{0}$ as being strictly zero and passing to a new lattice with size $r_{0}$. We then have a lattice gas consisting of two species $N$ and $\bar{N}$. Two $N$ (or two $\bar{N}$ ) particles can be in neighboring cells while a $N$ and a $\bar{N}$ cannot be first neighbors to each other. It is then intuitively clear that, when the numbers of $N$ and $\bar{N}$ in the lattice grow as temperature increases, this statistical repulsion between $N$ and $\bar{N}$ can only be accommodated if $N$ and $\bar{N}$ separate into different regions of space containing many cells.

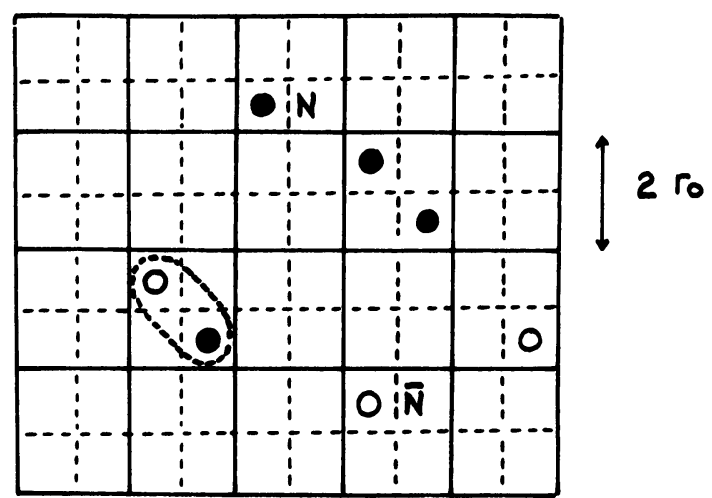

Fig. 5. A lattice space model of nucleon-antinucleon separation.

In fact, this model had been introduced independently by Widom and Rowlinson (1970) with quite different applications in mind and, recently, Ruelle (1971) has proved rigorously (in Ruelle's sense of the word) that the phase transition exists.

According to Widom and Rowlinson, the critical situation is obtained when the average number of particles $N$ or $\bar{N}$ per cell is one, which corresponds typically, with values of $r_{0}$ less than a fermi to a temperature of the order of a few hundred $\mathrm{MeV}$.

\section{A Second Model}

Another model has been proposed and investigated by several people (Ommes, 1969a, 
1969b, 1970, 1972; Aldrovandi and Caser, 1972; Cisneros, 1972). The idea is the following: one starts from a virial expansion of the free energy $F$ as a function of the nucleon and antinucleon densities $N$ and $\bar{N}$. In practice, it reads

$$
\begin{aligned}
F(T) & =F(\text { mesons, } T)+F_{0}(\text { free nucleons, } N, T)+F_{0}(\bar{N}, T)+ \\
& +a(N+\bar{N})+b N \bar{N}+c\left(N^{2}+\bar{N}^{2}\right) .
\end{aligned}
$$

The weakness of the treatment is to truncate the virial expansion to second-order terms. While this is known to preserve the critical behavior of the Van der Waals transition, it is nevertheless a strong assumption.

On the other hand, the virial coefficients $a, b, c$ can be explicitly computed by using the scattering amplitudes for particle interactions. Indeed, it is known that a second virial coefficient can be expressed in terms of the phase shifts by means of the BethUhlenbeck formula

$$
2^{d} \text { virial coefficient } \propto \sum_{\alpha} \int \frac{\mathrm{d} \delta_{\alpha}}{\mathrm{d} E} e^{-E / k T} \mathrm{~d} E
$$

so that the knowledge of the pion-nucleon and nucleon-nucleon phase shifts makes it possible to compute $a$ and $c$. The calculation of $b$ is more difficult but, according to Dashen et al. (1969), it can be cast into the form

$$
b \propto \sum_{n} \int\left\langle N \bar{N}\left|S^{+}\right| n\right\rangle \frac{\stackrel{\leftrightarrow}{\partial}}{\partial E}\langle n|S| N \bar{N}\rangle e^{-E / k T} \mathrm{~d} E,
$$

where $S$ is the collision matrix. In this expression, terms of the type $\langle N \bar{N}|S| N \bar{N}\rangle$ have been computed by using either the mesonic theory of nuclear forces or phenomenological potentials. Annihilation matrix elements of the type $\langle N \bar{N}|S|$ any number of pions > can be obtained from the statistical model for annihilation.

It is found in this way that $b$ is large and negative (leading essentially to a statistical

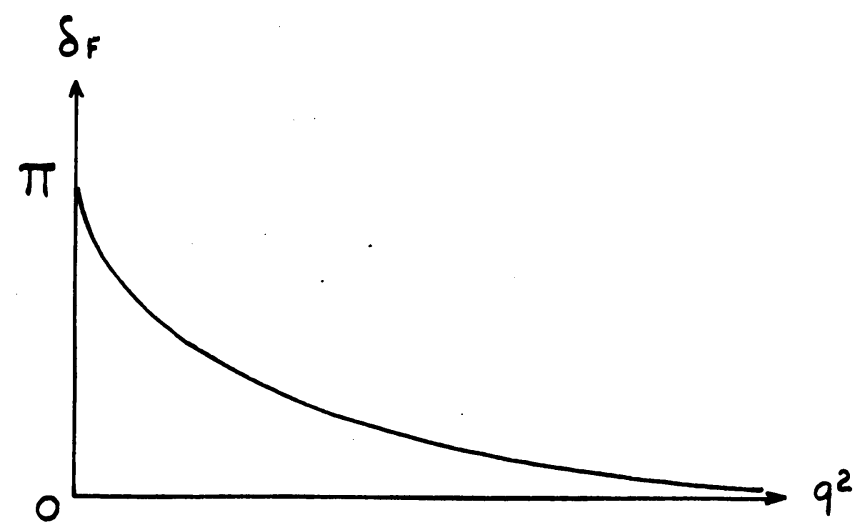

Fig. 6. A $S$-wave phase shift for nucleon-antinucleon sçattering. 
repulsion effect). This is due to the fact that, since there are bound states in all $S$-wave nucleon-antinucleon states, the corresponding phase shifts (or more technically, the Froissart phase shifts which take annihilation into account), decrease with the energy so that $\mathrm{d} \delta / \mathrm{d} E$ is negative for these states. This is the way the bound-state assumption enters into this calculation. (See Figure 6.) On the other hand, the mesons can be treated as elementary particles (as shown by Dashen et al. (1969), so that assumption (ii) (of Section 3) is also included in the independence of $F_{0}$ (mesons) upon $N$ and $\bar{N}$. Imposing the thermodynamical conditions

$$
\frac{\partial F}{\partial N}=0, \quad \frac{\partial F}{\partial \bar{N}}=0
$$

one finds again a phase transition with a critical temperature $T_{\mathrm{c}}$ of the order of 350 $\mathrm{MeV}$.

For the sake of experimental verification, let us note that it would be important to check whether or not, as is assumed here, the real part of the $S$-wave $N-\bar{N}$ scattering lengths are negative. This could be observed by measuring the sign of the level shifts in the protonium (i.e., the $p-\bar{p}$ ) atom.

We shall conclude this part of our report by saying that, although no rigorous proof of the separation effect has been really obtained, the convergence of these approaches gives strong enough support for its existence to warrant a serious investigation of its cosmological consequences.

\section{The Coalescence Effect}

Now we turn to the physical situation during the radiative period: we have to consider a system where matter and antimatter occupy different regions of space more or less uniformly. The general geometry of their distribution is of an emulsion and we shall call the scale of this emulsion $L$, i.e., any typical length. (See Figure 7.) Because of the short annihilation mean free path, matter and antimatter penetrate each other only in a small annihilation region along the boundary with width $h \ll L$. It is important to remember that everything is embedded inside thermal radiation.

It was noticed some years ago by Alfvén and Klein (1962) that annihilation produces pressure in such a system (this is what they called a Leidenfrost effect): indeed, annihilation along the boundary produces pions which decay either into gamma rays $\left(\pi^{0} \rightarrow \gamma+\gamma\right)$ or into electrons and positrons $\left(\pi^{ \pm} \rightarrow \mu^{ \pm} \rightarrow \mathrm{e}^{ \pm}\right)$forgetting about the neutrinos which are unimportant here. These high energy particles $\left(\gamma, \mathrm{e}^{ \pm}\right)$with small masses carry a large momentum and therefore exert a strong pressure upon the neighboring medium.

The question we shall ask now is the following: what is the general behavior of the fluid motions which are generated by the energy release and the pressures which are due to annihilation?

A first orientation can be obtained, using a thermodynamical argument which is due to P. G. de Gennes and A. Blandin. Let us assume that the system matter + anti- 
matter + radiation can be treated as a thermostat with temperature $T$. The system $\sigma$ made up by the high-energy gamma rays and $\mathrm{e}^{ \pm}$which are not yet thermalized is not in thermal equilibrium. According to a well-known theorem in thermodynamics, its free energy $F_{\sigma}$ must therefore tend to decrease. When computing this free energy $F_{\sigma}=E_{\sigma}-T S_{\sigma}$, one finds that, because of the presence of high-energy particles, the energy part $E_{\sigma}$ of $F_{\sigma}$ is larger than the entropy part, so that $F_{\sigma}$ is positive. On the other

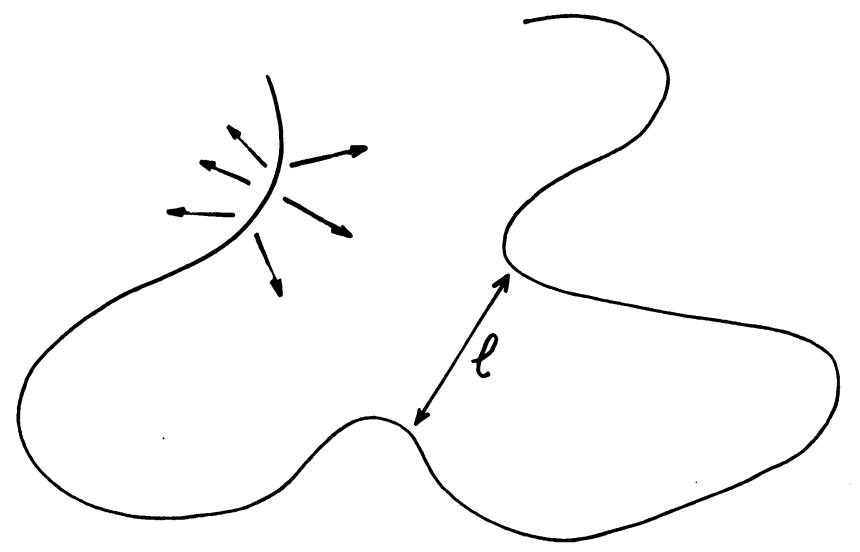

Fig. 7. An emulsion.

hand, the total number of particles in $\sigma$ is obviously proportional to the area $S$ of the matter-antimatter boundary $S$, so that

$$
F_{\sigma}=A S \text {. }
$$

Therefore $S$ must decrease. But since matter and antimatter fill up all space in equal amounts, this is possible only if $L$ increases. (It can be useful for comparison to notice that $F_{\sigma}$ has exactly the same form as a surface tension free energy.)

Therefore we are led to propose the following Ansatz: The coalescence effect; the scale $L$ of the emulsion increases with time as a side effect of the Leidenfrost mechan-

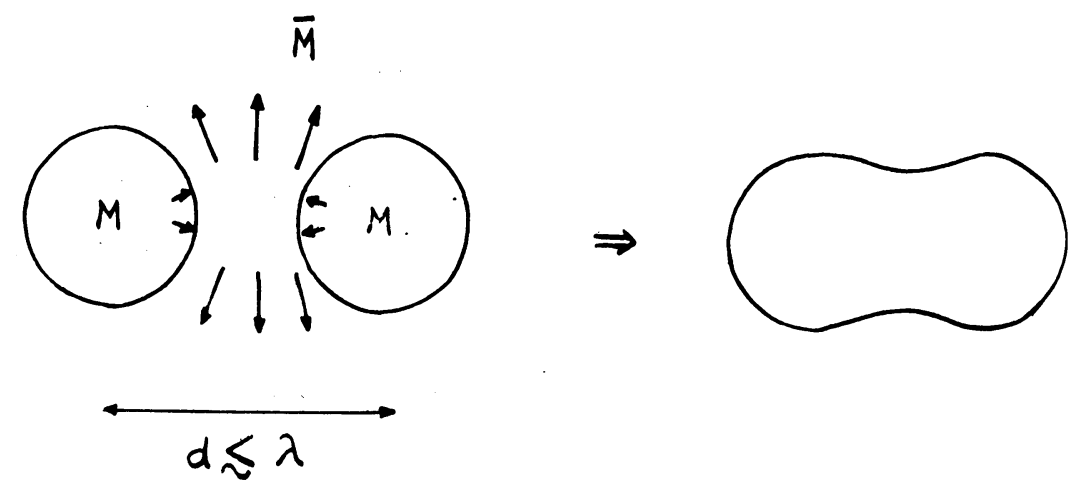

Fig. 8. The coalescence of two matter bubbles embedded in antimatter. 
ism, so that matter and antimatter are coalesced into larger and larger regions of space as time increases.

What was insufficient in the Blandin - de Gennes argument? It was to consider the system matter + antimatter + radiation as a thermostat, since precisely strong temperature gradients can be expected as part of the Leidenfrost effect. Therefore, we shall now review how the problem of coalescence can be tackled using the adequate techniques of hydrodynamics and what are the results of this approach.

\section{The Coalescence Effect: Hydrodynamics}

Here we follow the treatment reported in Ommès (1971b, 1971c).

Let us denote the local density of nucleons (or antinucleons) by $N(x, t)$ the fluid velocity by $\mathbf{v}$ (over distances larger than the mean free path for a thermal photon, matter + radiation can be considered as making only one fluid), the mass density (of matter + radiation) by $\varrho$, and the energy density for thermal radiation by $E$. An important quantity in the problem is the energy current $c \mathbf{J}(\mathbf{x}, t)$ carried by the annihilation products to a point $\mathbf{x}$. One has

$$
\mathbf{J}(\mathbf{x}) \propto \int_{S} \mathrm{~d} S_{0} \frac{\mathbf{x}-\mathbf{x}_{0}}{\left|\mathbf{x}-\mathbf{x}_{0}\right|^{3}} e^{-\chi\left(\mathbf{x}, \mathbf{x}_{0}\right)}
$$

where the total absorption $\chi$ is given by

$$
\chi=\int_{x_{0}}^{x} n \sigma \mathrm{d} l .
$$

Here, $\sigma$ is the cross section for interaction between an electron and high-energy photons (in practice $\sigma$ is 40 times the Thomson cross section). The integration in (13) is made along the boundary.*

The basic equations are, as usual, particle conservation, energy conservation and the equation of motion. Disregarding negligible terms they read

$$
\begin{aligned}
& (\partial N / \partial t)+\nabla(N v)=0, \\
& (\partial E / \partial t)+\nabla\left(J c-D \nabla E+\frac{4}{3} v E\right)=0, \\
& \varrho \frac{\mathrm{d} v}{\mathrm{~d} t}=-\nabla \frac{E}{3}+\frac{J}{\lambda_{\gamma}^{\text {h.e. }}} .
\end{aligned}
$$

The meaning of these equations is quite clear: in (16), the $J c$ term represents the heat source, the next term is conduction by diffusion and the last one conduction by convection. In (17) we notice that the radiative pressure is $E / 3$ and $J / \lambda_{\gamma}^{\text {h.e. }}\left(\right.$ with $\left.\lambda_{\gamma}^{\text {h.e. }}=(N \sigma)^{-1}\right)$ is the force exerted upon a unit volume of the fluid.

* When $k T>100 \mathrm{eV}, \chi$ is controlled by $\gamma+\gamma \rightarrow \mathrm{e}^{+} \mathrm{e}^{-}$(as pointed out by G. Steigman). 
Of particular importance are the boundary conditions which must be added to these equations in order to describe the behavior of the particles along the matter-antimatter boundary. Here the characteristic parameter is $\lambda_{0}$, the mean free path for thermal photons. For distances to the boundary smaller than $\lambda_{0}$, the hydrodynamical approximation is not valid and one must describe the propagation and thermalization of the particles by using kinetic theory. The essential result which is found in this way is the existence of a discontinuity pressure $\delta p$ across the boundary which is related to the curvature of the boundary by

$$
\delta p=2 \alpha / R
$$

This relation is identical with the Laplace-Kelvin condition which holds when there is a surface tension with coefficient $\alpha$. The value of $\alpha$ is given by

where

$$
\alpha=\frac{2}{3} J(0) \lambda,
$$

$$
\lambda=\operatorname{Max}\left(\lambda_{0}, \lambda_{\gamma}^{\text {h.e. }}\right) \text {. }
$$

Here $J(0)$ is essentially given by the rate of annihilation along the boundary which is itself controlled by diffusion.

The origin of the discontinuity $\delta p$ can be easily visualized: Along the boundary, there are the same number of high-energy particles going in each direction, so that they carry the same momentum into matter and antimatter. Because of the curvature, the flux of momentum per unit area (i.e. pressure) is larger in a convex region.

Because of the formal identity of the boundary condition (18) with the effect of a surface tension and because of the large value of $\alpha$, coalescence will result. Dimensional arguments show that the size of the emulsion $L$ will increase with time according to the rule

$$
L^{3}=\frac{\alpha}{\varrho} t^{2}
$$

Apart from these results, which are the most important, some significant properties of (15) to (17) are also known:

(i) There is no hydrostatic solution, except for a plane or spherical boundary. The plane boundary is hydrodynamically stable.

(ii) The regime of convective motions can be investigated by freezing the motion of the boundary. It is found that, after a short transient period of the order of $L / c_{\mathrm{s}}$ (where $c_{\mathrm{s}}$ is the sound velocity), a balance is established between the force $J / \lambda_{\gamma}^{\text {h.e. }}$ and the radiative pressure. (The physical explanation is the following: gamma rays push electrons which drag radiation and build up a pressure gradient.) In these conditions, heat transfer, as governed by (16) determines a convective motion. The effect of this motion has been found in a special case (two bubbles of matter embedded inside antimatter) to produce further coalescence. However, these convective velocities are much smaller than the ones given by (21). 
As a conclusion, the coalescence effect will most probably be well established very soon. [Though we point out that (18), a relatively new result, needs further confirmation.] We shall therefore now consider its consequences in cosmology.

\section{Cosmological Consequences}

Applying these ideas to the evolution of the Universe as described by the previously introduced model, we find a new succession of periods as shown in Table II. [The entries in this table have been compiled from Ommes (1971a), Schatzman (1970), Kundt (1971) but include modifications due to an effect pointed out by G. Steigman in a private communication and also some results derived from Equations (18) and (19).]

TABLE II

Evolutionary periods in our model

\begin{tabular}{llll}
\hline Temperature $T$ & $350 \mathrm{MeV}$ & $40 \mathrm{keV}$ & $3000 \mathrm{~K}$ \\
\hline Time $t$ & $10^{-5} \mathrm{~s}$ & $\sim 15 \mathrm{~min}$ & $10^{6} \mathrm{yr}$ \\
\hline $\begin{array}{l}\text { Emulsion size }(L) \\
(\mathrm{cm})\end{array}$ & $10^{-4} \mathrm{~cm}$ & $\sim 10^{3.5} \mathrm{~cm}$ & $\sim 10^{22} \mathrm{~cm}$ \\
\hline Period & separation & annihilation & coalescence \\
\hline
\end{tabular}

We shall briefly describe the main features of this evolution.

(i) The separation of the two phases takes place above the critical temperature $T_{\mathrm{c}}$. The size of the emulsion near $T_{\mathrm{c}}$ can be computed by standard techniques and is found to be given by $L=[D t]^{1 / 2}$ where $D$ is the diffusion coefficient for the baryonic number. Any of the conventional formulas for $D: D \simeq(\mathrm{k} T / m) \mathscr{T} \simeq c \lambda$, give the same results and the value $L_{\mathrm{c}}=10^{-4} \mathrm{~cm}$. Somewhat above $T_{\mathrm{c}}$, the coefficient $\eta$, as computed from the baryonic density, is of the order of

$$
\eta_{c} \simeq 7\left(\frac{T-T_{c}}{T_{c}}\right)^{1 / 2}
$$

so that $\eta_{\mathrm{c}}$ is in fact of the order of unity $50 \mathrm{MeV}$ above $T_{\mathrm{c}}$. It tends to decrease when $T$ approaches $T_{\mathrm{c}}$ so that, for the sake of definiteness, we shall consider that the end of the separation period takes place slightly above $T_{\mathrm{c}}$ with a value of $\eta_{\mathrm{c}}$ not very different from unity.

(ii) The annihilation period is characterized by a strong mixing of nucleons with antinucleons which leads to a large relative amount of annihilation. The size of the emulsion and the value of $\eta$ at any given time can be known explicitly: Since particles are lost by diffusion, one has

$$
\frac{\mathrm{d} N}{\mathrm{~d} t} \simeq 2 D \Delta N \simeq 2 D \frac{N}{L^{2}}
$$


(the factor 2 is for antiparticles entering). So that (taking expansion into account) one has

$$
\left|\log \frac{\eta}{\eta_{c}}\right| \simeq|\log \eta|=\frac{2 D t}{L^{2}} .
$$

On the other hand, this same quantity $\eta$ can be computed by saying that, in a given region of size $L$, what baryons remain after annihilation were the fluctuations initially present in the same region. If fluctuations were as usual, one would find:

$$
\eta=\eta_{c}\left(\frac{L_{c} T_{c}}{L T}\right)^{3 / 2}
$$

the factor $T_{0} / T$ being due to expansion. However, because of the correlations in the initial fluctuation of the baryon number (there is no volume fluctuation of the baryon number, only surface fluctuations near the boundary of the emulsion with size $L$ ), one has

$$
\eta=\eta_{c}\left(\frac{L_{c} T_{c}}{L T}\right)^{2}
$$

Taken together (23) and (24) give a determination of $\eta$ and $L$ during the annihilation period.

As pointed out by $\mathrm{G}$. Steigman, the value of $D$ during the leptonic period is mainly given by neutron diffusion (the large neutron mean free path being controlled by electron-neutron scattering). Annihilation proceeds rapidly as long as weak interactions maintain a balance between protons and neutrons (e.g., $v+p \rightleftarrows \mathrm{e}^{+}+n$ ). Near $k T \simeq 3.5 \mathrm{MeV}$, this effect of weak interactions stops and $\eta$ is found to be of the order of $10^{-8}$.

From $3.5 \mathrm{MeV}$ to $50 \mathrm{keV}$, the system is rather quiescent. The diffusion of protons is slow because of plasma effects, and coalescence, which is beginning to take place, is not yet very effective. From $50 \mathrm{keV}$ to $3000 \mathrm{~K}$ coalescence becomes very active and brings together large quantities of matter rapidly, the typical size of the emulsion being given by (21). It turns out that the relative amount of annihilation is small during this last period.

\section{Conclusion and Perspectives}

Coalescence stops at the time of recombination because of the rapid decrease in the sound velocity which would make coalescent motions supersonic.

The details of these physical processes can be rather intricate and they are presently being investigated by S. Caser, J. L. Puget, G. Valladas and myself. We cannot yet give final numbers with complete confidence until this investigation is completed, which will take some time. However I shall quote a few preliminary results which are subject to change: $\eta$ is slightly smaller than $10^{-8}$, the mass $M=\varrho L^{3}$ is of the order of $10^{11}$ solar masses or more (i.e., a galactic mass or a cluster mass) and there is no primeval helium because of the neutron loss during the leptonic period. 
It is gratifying that the simplest model of the Universe, together with a few well-defined effects which are within the reach of theoretical investigation, leads to an evolution which can be followed in some detail. The quantitative conclusions, as to the amount of matter in the Universe and the galactic masses seem to agree rather well with observation.

The main problems will now be to link these ideas with astrophysics and we list here a few of them:

When does the gravitational collapse of galaxies take place?

How important is the role of annihilation pressure in gravitational collapse ? Can it take place through the effect of a magnetic field stopping the electrons?

More generally, what are the plasma effects (e.g., magnetic instabilities) when matter and antimatter are in contact?

What are the observable effects of coalescence velocities (e.g., turbulence, angular momentum (Puget and Stecker, 1972).

Once galaxies are formed, how frequently do they collide? Are such collisions responsible for the angular momentum of galaxies, as a simple calculation suggests?

Is the clustering of galaxies associated with the correlations inside matter which are due to the geometry of the emulsion?

Does annihilation in the later stages sufficiently distort the spectrum of thermal radiation or of the X-ray background to be detectable (Stecker et al., 1971; Sunyaev and Zeldovich, 1970)?

Should we revive the antimatter model for quasars: they could be made of matter (of a nearly galactic size), initially trapped within antimatter? Energy and age requirements are easily met.

What happens to a cloud of antimatter inside a galaxy? We know that annihilation leads to a strong viscosity: does the object fall into the center of the galaxy? Or is it ejected?

Where and when are helium and deuterium synthesized? (See some of the comments by A. G. W. Cameron in this volume.)

And last but not least, can we find an observational test to make sure whether antimatter does or does not exist?

\section{References}

Aldrovandi, R. and Caser, S.: 1972, Nucl. Phys. B38, 593.

Alfvén, H. and Klein, O.: 1962, Arkiv Fysik 23, 187.

Cisneros, A.: 1972, California Institute of Technology Thesis (to be published).

Dashen, R., Ma, S., and Bernstein, H. J.: 1969, Phys. Rev. 187, 345.

Kundt, W.: 1971, A Survey of Cosmology, Springer Tracts, Heidelber 5.

Omnès, R.: 1969a, Phys. Rev. Letters 23, 38.

Omnès, R.: 1969b, Ann. Phys. Paris 4, 515.

Omnès, R.: 1970, Phys. Rev. 1, 723.

Omnès, R.: 1971a, Astron. Astrophys. 10, 228.

Omnès, R.: 1971b, Astron. Astrophys. 11, 450.

Omnès. R.: 1971c, Astron. Astrophys. 15, 275.

Omnès, R.: 1972, Phys. Reports 3C, No. 1.

Puget, J. L. and Stecker, F. W.: 197? (to be published). 
Ruelle, D.: 1971, Phys. Rev. Letters 27, $104 C$.

Schatzman, E.: 1970, CERN Report.

Stecker, F. W., Morgan Jr., D. L., and Bredekamp, J.: 1971, Phys. Rev. Letters 27, 1469.

Sunyaev, R. A. and Zeldovich, Ya. B.: 1970, Comments Astrophys. Space Phys. 11, 66.

Widom, B. and Rowlinson, J. S.: 1970, J. Chem. Phys. 52, 1670. 